\title{
Theory of Creeping Waves in Acoustics and Their Experimental Demonstration
}

\author{
In memoriam Arnold Sommerfeld *
}

\author{
W. G. Neubauer
}

Naval Research Laboratory, Washington, D.C. 20390

$$
\text { P. UGINČIUS }
$$

U.S. Naval Weapons Laboratory, Dahlgren, Va. 22448

\section{H. ÜBeraLL}

The Catholic University of America, Washington, D.C. 20017

(Z. Naturforsch. 24 a, 691-700 [1969]; received 3 February 1969)

\begin{abstract}
The theory of creeping waves is developed by applying the Sommerfeld-Watson transformation on the normal-mode solution for acoustic scattering from an infinite elastic cylinder. Two types of radiating, circumferential waves emerge: (1) highly attenuated "Franz-type" waves with subsonic speeds, and (2) supersonic "Rayleigh-type" waves which are only slightly attenuated. The experimental study of these waves for an aluminium cylinder is accomplished by two independent methods: (1) time measurements of sequential pulses, and (2) direct visualization by Schlieren techniques. Measured velocities, attenuations, critical angles, and scattering cross sections are compared with theoretical calculations.
\end{abstract}

Among the multitude of classical problems which A. SoMmerfeld ${ }^{1}$ considered, the one which is most relevant to the subject matter of this paper is the problem of propagation of radio waves around the earth. He solved it by using a mathematical method which is now known as the "Sommerfeld-Watson transformation", and which we shall employ here also. VAN Der PoL and Bremmer ${ }^{2}$ addressed themselves to the same problem, and FrANz ${ }^{3-7}$ advanced the theory considerably by applying it to diffraction of electromagnetic waves around conducting cylinders and spheres. It was he who coined the term "creeping wave", (Kriechwelle), to describe the phenomena of circumferentially propagating waves.

Only recently came the realization that the same creeping-wave theory furnishes a most useful description for analyzing acoustic scattering as well. The impetus for this was given by the experimental discovery by BARNARD and MCKINNEY 8 that a single underwater sound pulse which is incident on

* The authors wish to thank the editors of the Zeitschrift für Naturforschung for the privilege of dedicating this paper to the memory of ARNold Sommerfeld's hunredth birthday, Dec. 5, 1968.

1 A. Sommerfeld, Partial Differential Equations in Physics, Academic Press, New York-London 1964. See particuiarly the appendix after chapter 6 .

2 B. van Der Pol and H. Bremmer, Phil. Mag. 24, 141, 825 [1937].

3 W. Franz and K. Deppermann, Ann. Phys. Leipzig 10, 361 [1952]. a scatterer will produce a series of multiple echo returns. Since then a number of other experimenters 9 have corroborated the basic idea of this paper: that the physical mechanism for acoustic scattering consists of a superposition of continuously radiating circumferential waves. The creeping-wave theory furnishes this physical picture in a most natural way, via the Sommerfeld-Watson transformation, whereas in the classical "normal mode solution" (an infinite-series expansion in terms of separable eigenfunctions of the wave equation) this interpretation is hidden. Consistent also with the truism that of all possible mathematical descriptions for a given physical phenomenon the most efficient one will be the one which resembles the physics of that phenomenon most closely, we find that the creeping-wave solution is much more rapidly convergent than the normal-mode solution. Acoustic scattering is particularly suited for the experimental study of creeping waves, (better than electromagnetic scattering),

4 W. Franz, Z. Naturforsch. 9 a, 705 [1954].

5 W. Franz and P. Beckmann, IRE Trans. Antennas and Propagation AP-4, 203 [1956].

6 P. Beckmann and W. Franz, Z. Naturforsch. 12 a, 257 [1957].

7 W. Franz, Theorie der Beugung Elektromagnetischer Wellen, Springer-Verlag, Berlin 1957.

8 G. R. Barnard and C. M. McKinney, J. Acoust. Soc. Am. 33, 226 [1961].

9 See the bibliographies in references 11 and 13 . 
because of their slow propagation speeds and, more important, because they can be observed directly by the use of pulsed signals.

The theory of creeping waves has been applied by the present authors to various problems in acoustic scattering from rigid, soft, or elastic cylinders or cylindrical shells using both continuous and pulsed waves ${ }^{10-16}$. In this paper, part A, we present the creeping-wave analysis of acoustic scattering by an infinite elastic cylinder immersed in a fluid. Two types of circumferential waves emerge: (1) highly attenuated "Franz-type" waves which are slower than $c$ - the speed of free acoustic waves in the liquid; and (2) very slightly attenuated "Rayleigh-type" waves which are faster than $c$. The former are the original creeping waves first identified by Franz, and depend mainly on the geometry of the scatterer, whereas the latter owe their existence solely to the scatterer's elastic properties. In part B we present the experimental verification of the theory for an aluminium cylinder, and visualization of the creeping waves by Schlieren methods. This is the first time that both the Franz-type and Rayleigh-type waves were definitely identified in an experiment and correlated with theory. A more detailed description of this experiment can be found in Refs. 17 and 18 .

\section{Part A: Theory}

\section{The Normal-Mode Solution}

Figure 1 shows the geometry of the problem: a plane wave $\exp i\left(k_{1} x-\omega t\right)$ is incident from the negative $x$-axis on an elastic cylinder with radius $a$, density $\varrho_{2}$, and Lamé constants $\lambda, \mu$. The surrounding medium is an infinite homogeneous fluid with density $\varrho_{1}$, in which the speed of propagation for the acoustic wave is

$$
c_{1}=\omega / k_{1}
$$

whereas inside the cylinder the longitudinal (compressional) and transverse (shear) modes have the

10 R. D. Doolittle and H. Überall, J. Acoust. Soc. Am. 39, 272 [1966].

11 H. Überall, R. D. Doolittle, and J. V. McNicholas, J. Acoust. Soc. Am. 39, 564 [1966].

12 R. D. Doolittle, J. V. McNicholas, H. Úberall, and P. Uginčius, J. Acoust. Soc. Am. 42, 522(L) [1967].

13 R. D. Doolittle, H. Úberall, and P. Uginčius, J. Acoust. Soc. Am. 43, 1 [1968].

14 P. Uginčius and H. Überall, J. Acoust. Soc. Am. 43 , 1025 [1968].

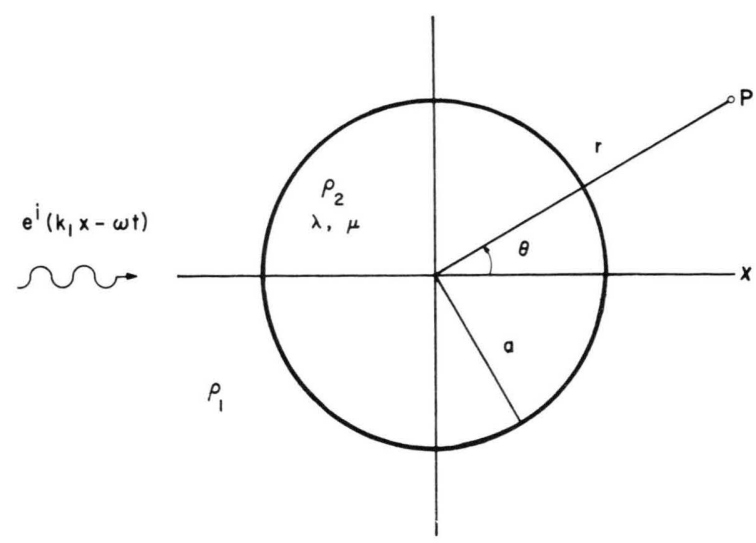

Fig. 1. Scattering Geometry.

respective speeds

$$
c_{1}=\left[(\lambda+2 \mu) / \varrho_{2}\right]^{1 / 2} ; \quad c_{\mathrm{t}}=\left(\mu / \varrho_{2}\right)^{1 / 2} .
$$

The total acoustic pressure at the general observation point $P(r, \theta)$ is obtained in the usual way, by subjecting the general separable solution in terms of the cylinder eigenfunctions to the elastic boundary conditions at $r=a$. After suppressing the time dependence $\exp (-i \omega t)$ the result is (see $\left.{ }^{10.16}\right)$ :

$$
p=p_{\text {inc }}+p_{\mathrm{sc}},
$$

where the incident and scattered pressures are given respectively by

$$
\begin{gathered}
p_{\text {inc }}=\sum_{n=0}^{\infty} i^{n}\left(2-\delta_{n 0}\right) J_{n}\left(k_{1} r\right) \cos (n \theta), \\
p_{\mathrm{se}}=\sum_{n=0}^{\infty} i^{n}\left(2-\delta_{n 0}\right) \frac{B_{n}}{D_{n}} H_{n}^{(1)}\left(k_{1} r\right) \cos (n \theta),
\end{gathered}
$$

and where the expansion coefficients in Eq. $(3 \mathrm{c})$ are found to be

$$
B_{n}=\left|\begin{array}{lll}
\beta_{1} & \alpha_{12} & \alpha_{13} \\
\beta_{2} & \alpha_{22} & \alpha_{23} \\
0 & \alpha_{32} & \alpha_{33}
\end{array}\right| ; \quad D_{n}=\begin{array}{ccc}
\alpha_{11} & \alpha_{12} & \alpha_{13} \\
\alpha_{21} & \alpha_{22} & \alpha_{23} \\
0 & \alpha_{32} & \alpha_{33}
\end{array} .
$$

With the introduction of the dimensionless parameters

$$
x_{i}=a k_{i}=a \omega / c_{i}, \quad(i=1, l, t)
$$

15 J. V. McNicholas, H. Überall, and K. Choate, J. Acoust. Soc. Am. 44, 752 [1968].

16 P. Ugrnčrus, Ph. D. thesis, Phys. Dept., The Catholic Univ. of America, 1968; and U. S. Naval Weapons Lab. Tech. Rept. No. 2128, Feb. 1968, Dahlgren, Va. 22448. 17 W. G. Neubauer, J. Acoust. Soc. Am. 44, 298 (L) [1968].

18 W. G. Neubauer, Ph. D. thesis, Physics Dept., The Catholic Univ. of America, 1968; and J. Acoust. Soc. Am., to be published. 
the elements of these determinants are given by 19 (primes indicate differentiation with respect to the argument):

$$
\begin{aligned}
\beta_{1} & =\left(\varrho_{1} / \varrho_{2}\right) x_{t}^{2} J_{n}\left(x_{1}\right), \\
\beta_{2} & =x_{1} J_{n}^{\prime}\left(x_{1}\right) \\
\alpha_{11} & =-\left(\varrho_{1} / \varrho_{2}\right) x_{t}^{2} H_{n}^{(1)}\left(x_{1}\right), \\
\alpha_{12} & =-2 x_{1} J_{n}^{\prime}\left(x_{1}\right)+\left(2 n^{2}-x_{t}^{2}\right) J_{n}\left(x_{1}\right), \\
\alpha_{13} & =2 n\left[J_{n}\left(x_{\mathrm{t}}\right)-x_{\mathrm{t}} J_{n}^{\prime}\left(x_{\mathrm{t}}\right)\right] \\
\alpha_{21} & =-x_{1} H_{n}^{(1)^{\prime}}\left(x_{1}\right), \\
\alpha_{22} & =-x_{1} J_{n}^{\prime}\left(x_{1}\right), \\
\alpha_{23} & =n J_{n}\left(x_{\mathrm{t}}\right) ; \\
\alpha_{32} & =2 n\left[x_{1} J_{n}^{\prime}\left(x_{1}\right)-J_{n}\left(x_{1}\right)\right], \\
\alpha_{33} & =2 x_{\mathrm{t}} J_{n}^{\prime}\left(x_{\mathrm{t}}\right)+\left(x_{t}^{2}-2 n^{2}\right) J_{n}\left(x_{\mathrm{t}}\right) .
\end{aligned}
$$

A quantity of experimental interest is the differential scattering cross section defined by

$$
\mathrm{d} \sigma / \mathrm{d} \theta=\lim _{r \rightarrow \infty} r\left|p_{\mathrm{sc}} / p_{\text {inc }}\right|^{2} .
$$

By using Hankel's asymptotic expansion for large argument

$$
H_{n}^{(1)}(\varrho) \cong(2 / \pi \varrho)^{1 / 2} \exp i\left[\varrho-(\pi / 2)\left(n+\frac{1}{2}\right)\right]
$$

for the scattered pressure in Eq. (3c), we arrive at the following expression for the cross section:

$$
\frac{\mathrm{d} \sigma}{\mathrm{d} \theta}=\frac{2}{\pi k_{1}}\left|\sum_{n=0}^{\infty}\left(2-\delta_{n 0}\right) \frac{B_{n}}{D_{n}} \cos (n \theta)\right|^{2} .
$$

In principle this equation could be summed to any desired degree of accuracy. However, for large values of $k a\left(x_{1} \gtrsim 1\right)$ Eq. (9) is limited by its very slow convergence. For example, Sommerfeld ([1], p.282), in a similar problem finds that a normal-mode series like that of Eq. (9) would require more than 1000 terms before it would start to converge.

\section{The Creeping-Wave Solution}

We now reformulate the normal-mode solution of the preceding section via the Sommerfeld-Watson transformation ${ }^{1,20}$. This will not only improve the convergence of the solution but, more important, will furnish the physical picture of the circumferential creeping waves. The Sommerfeld-Watson

19 These differ from the corresponding elements in Ref. 10 and 13 by the overall factor of $\mu$, thereby making them dimensionless. See also Ref. ${ }^{14}$ or ${ }^{16}$ where the correctly nondimensionalized elements are given for the more general $6 \times 6$ determinants for a cylindrical shell. transformation may be written in the form

$$
\sum_{n=0}^{\infty}\left(2-\delta_{n 0}\right) f_{n}=i P \int_{\mathrm{C}} \frac{\mathrm{d} v}{\sin \pi v} e^{-i \pi v} f(v),
$$

where $\mathrm{C}$ is a contour which passes through the origin $v=0$, surrounding the positive real axis in clockwise sense, and excludes all poles of the function $f(v)$. The Neumann factor $2-\delta_{n 0}$ requires that the principal value be taken for the integration through the origin. The next step is to transform the contour $\mathrm{C}$ (which encloses all the zeroes of $\sin \pi v$ and no poles of $f(v)$ ) into a different contour which surrounds all poles of $f(v)$. To do this one must have an a priori knowledge of the complex zeroes of the determinant $D_{n} \rightarrow D(v)$, because applying the transformation (10) to Eq. (3c) we see that the only poles of the function $f(v)$ are those zeroes, all other cylinder functions which appear in $B_{n}$ and $H_{n}^{(1)}$ being entire functions in the complex $v$-plane of their order.

The determinant $D(v)$ is too complicated to yield any information about its roots by analytical methods. For special cases, however, it degenerates into simpler forms for which the asymptotic zeroes are well known. Thus, for example, for rigid $(\mu \rightarrow \infty)$ and soft $(\mu \rightarrow 0)$ cylinders it is found 10 that they are the roots of $H_{v}^{(1) \prime}\left(x_{1}\right)$ and of $H_{v}^{(1)}\left(x_{1}\right)$ respectively. For large $x_{1}$ these roots are known from the work of Sommerfeld ${ }^{1}$ and Franz ${ }^{4}$. There are infinitely many of them and they all lie in the first quadrant of the $v$-plane on a line which when extrapolated intersects the real axis at $v \cong x_{1}$.

We have written a computer program to find the zeroes of the determinant $D(v), \mathrm{Eq}$. (4), numerically (see ${ }^{16}$ ). Essentially it is a Newton-Raphson method generalized to the complex plane, which converges quite rapidly if the initial estimate is fairly good. To make sure that no zeros are overlooked we also made use of the "Principle of the Argument"

$$
2 \pi(Z-P)=\Delta_{\mathrm{C}} \operatorname{Arg} D(v),
$$

which states that the number of zeroes $Z$ minus the number of poles $P$ (with their multiplicities taken into account) inside any closed contour $\mathrm{C}$ is equal to the net change (divided by $2 \pi$ ) of the argument of $D(v)$ in a complete traversal of C. With $P=0$

20 G. N. Watson, Proc. Roy. Soc. London A59, 83, 546 [1919]. 
Eq. (11) enabled us to determine unambiguously the number of zeroes in any given region of the complex plane. The results for an aluminium cylinder $\left(\lambda=6.1 \cdot 10^{11} \mathrm{dyn} / \mathrm{cm}^{2}, \mu=2.5 \cdot 10^{11} \mathrm{dyn} / \mathrm{cm}^{2}\right.$, $\left.\varrho_{2}=2.7 \mathrm{gm} / \mathrm{cm}^{3}\right)$ at $x_{1}=k_{1} a=5$ are shown in Fig. 2. We find two sets of zeroes: (1) the set labelled $\mathrm{F}$ which lies entirely in in the first quadrant, and which differs numerically very little from the "rigid" Franz-type zeroes - the roots of $H_{v}^{(1) \prime}\left(x_{1}\right)=0$; and (2) the set labelled $R$ which starts out with two zeroes close to the positive real axis, and continues into the second quadrant approaching asymptotically the negative real axis. They seem to coalesce

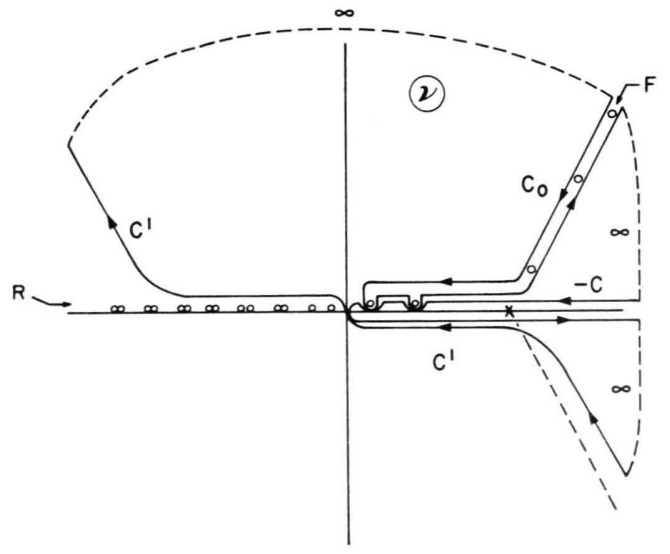

Fig. 2. Contours for the Sommerfeld-Watson Transformation. The circles show the Franz $(\mathrm{F})$ and Rayleigh-type $(\mathrm{R})$ zeroes of $D(v)$ for an aluminium cylinder with $k_{1} a=5$.

pairwise into the negative integers. This second set of Rayleigh-type zeroes is absent for either rigid or soft cylinders, and their existence, therefore, must be attributed to the elastic properties of the scatterer. We also find that there may be zeroes in the third quadrant; however, they were difficult to locate with the present numerical program. Fortunately, as will ke seen below, only first-quadrant zeroes close to the real axis can contribute in the theory. All these zeroes are functions of $x_{1}$. The behaviour of the Franz-type zeroes vs. $x_{1}$ is the same as that deduced from their asymptotic formulas (see ${ }^{1}$ or ${ }^{4}$ ). The line of Rayleigh-type zeroes, however, seems to be "pulled" into the first quadrant with increasing $x_{1}$, so that it appears that for $x_{1} \rightarrow \infty$ there may be an infinite number of them in the first quadrant (see Fig. 5 in Ref. ${ }^{14}$ ). For a cylindrical shell (14 and $\left.{ }^{16}\right)$ the analogous function $D(v)$ is a six-by-six determinant. There we find the zeroes are qualitatively the same as in Fig. 2 with the exception of an additional set in the fourth quadrant.

Figure 2 also shows the transformation of the original contour C in the Sommerfeld-Watson integral (10). This is done with the additional contours $\mathrm{C}_{0}$ and $\mathrm{C}^{\prime}$ closed up at $\infty$ in such a way that the new closed contour $\mathrm{C}^{\prime}+\mathrm{C}_{\infty}+\mathrm{C}_{0}+\mathrm{C}_{\infty}-\mathrm{C}+\mathrm{C}_{\infty}$ does not include any poles, so that the integral in Eq. (10) over this contour must vanish. The peculiar shape of $\mathrm{C}^{\prime}$ is dictated by the requirement (see below the discussion after Eq. (22)) that no secondquadrant poles should be included, and by the fact, which Franz ${ }^{7}$ has shown, that the integral over $\mathrm{C}_{\infty}$ to the left of the dashed line in the fourth quadrant (which is the reflection of the F-line) would not converge. Vanishing of the integral over the portions $\mathrm{C}_{\infty}$ shown in Fig. 2 can be readily established. The transformation (10) applied on Eqs. (3) then yields for the total pressure

$$
\begin{gathered}
p=p_{\mathrm{I}}+p_{\mathrm{II}} \\
p_{\mathrm{I}}=i \int_{\mathrm{C}_{0}} \frac{\mathrm{d} v}{\sin \pi v} e^{-i v \pi / 2} \cos (v \theta) H_{v}^{(1)}\left(k_{1} r\right) \frac{B(v)}{D(v)} \\
p_{\mathrm{II}}=i P \int_{\mathrm{C}^{\prime}} \frac{\mathrm{d} v}{\sin \pi v} e^{-i v \pi / 2} \cos (v \theta) \\
\times \frac{J_{v}\left(k_{1} r\right) D(v)+H_{v}^{(1)}\left(k_{1} r\right) B(v)}{D(v)} .
\end{gathered}
$$

The "background" integral $p_{\text {II }}$ of Eq. (12c) is absent for either soft or rigid cylinders. For an elastic (aluminium) cylinder we have shown 13,14 that it is negligible (except maybe at some critical angles) by comparing results evaluated without that integral with the exact normal-mode calculations of FARAN ${ }^{21}$. We shall therefore neglect it. Note that the incident pressure which is proportional to $J_{v}\left(k_{1} r\right)$ seems to have dropped out of Eq. (12 b). We shall see below that this is not really so, but that it can be recovered by a saddle-point integration.

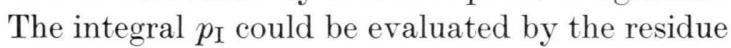
method, but Franz ${ }^{7}$ has shown that the resulting residue series would not converge in general, the reason being that $p_{\text {I }}$ also contains the geometrically reflected wave. That wave can be separated out of $(12 b)$ by writting for $\cos (v \theta)$ the identity

$\cos (v \theta)=e^{i \pi v} \cos v(\pi-\theta)-i e^{i v(\pi-\theta)} \sin (\pi v)$.

The last term cancels the $\sin (\pi v)$ in the denominator of Eq. (12b). This results in an integral which has

21 J. J. Farax, J. Acoust. Soc. Am. 23, 405 [1951]. 
been shown ${ }^{13}$ to represent the geometrically reflected wave. One attempts to evaluate it (for large $x_{1}$ ) by the saddle-point method. Using Hankel's asymptotic expansion, Eq. (8), for $H_{v}^{(1)}$ in Eq. (12 b), and Debye's asymptotic expansion

$$
\begin{aligned}
& H_{\nu}^{\left(\frac{1}{2}\right)}(x) \cong(2 / \pi x \sin \alpha)^{1 / 2} e^{ \pm i[x(\sin \alpha-\alpha \cos \alpha)-\pi / 4]}, \\
& v \equiv x \cos \alpha,
\end{aligned}
$$

for the first-column elements of $B(v)$ and $D(v)$, we have shown 13,16 that the saddle point is located at $\alpha_{\mathrm{s}}=\theta / 2$, or

$$
v_{\mathrm{S}}=x_{1} \cos (\theta / 2) .
$$

Figure 2 reveals that at the "critical angles"

$$
\theta_{k}^{*}=2 \cos ^{-1}\left(\operatorname{Re} v_{k} / x_{1}\right),
$$

where $v_{k}$ is any first-quadrant Rayleigh pole, the saddle point (15) will lie directly under a pole. This would mean that the resulting saddle-point evaluation for the geometrically reflected wave would be very inaccurate, because we would be unable to distort the contour $\mathrm{C}_{0}$ in order to make it pass through the saddle point without coming too close to a pole of the integrand. (We have shown that the path of steepest descent must go through the saddle point at an angle of $3 \pi / 4$ with the real axis). To overeome this difficulty we break up the contour $\mathrm{C}_{0}$ of Fig. 2 into the two separate contours $\mathrm{C}_{1}$ and $\mathrm{C}_{2}$, as shown in Fig. 3, before the separation of the geometric term by Eq. (13). We then evaluate the integral (12 b) by the Residue Theorem in its "un-

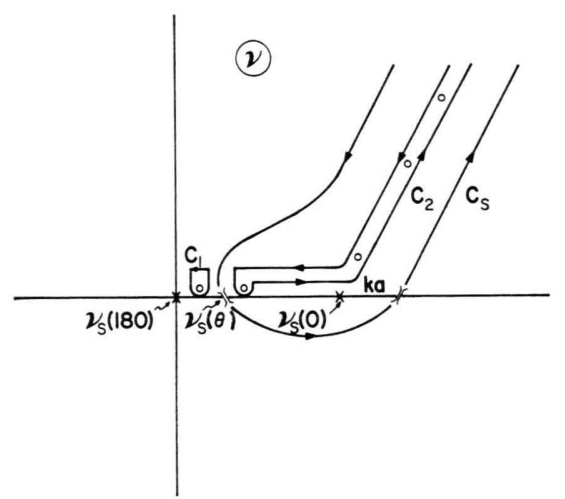

Fig. 3. Contours for Separation of the Geometrically Reflected Wave.

separated form" (leaving $\cos v \theta$ unaltered) over the contour $\mathrm{C}_{1}$, and in the "separated form" (rewriting $\cos v \theta$ by Eq. (13)) over the contour $\mathrm{C}_{2}$. These two residue series will yield the creeping waves. The geometrically reflected wave can be evaluated by the saddle-point method over the contour $\mathrm{C}_{\mathrm{s}}$ as outlined above. (Another saddle point is present which is shown schematically in Fig. 3 on the real axis to the right of $x_{1}$. Franz ${ }^{5,7}$ has shown that this restores the contribution of the incident wave, but since we are only interested in the scattered wave, we shall ignore it here.)

The results are:

$p_{\mathrm{I}}=p_{\mathrm{c}}+p_{\mathrm{g}} ;$

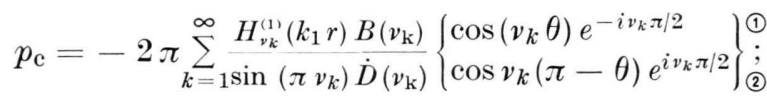

$p_{\mathrm{g}} \cong-[a \sin (\theta / 2) / 2 r] R\left(v_{\mathrm{s}}\right) e^{i k_{1}[r-2 a \sin \theta / 2]}$.

In the summation of Eq. (17b) $\dot{D}\left(v_{k}\right)=\partial D / \partial v$ evaluated at $v=v_{k}$; the forms (1) and (2) must be used when

$\operatorname{Re}\left(v_{k}\right)<x_{1} \cos (\theta / 2):$ (1) Unseparated Form;

$\operatorname{Re}\left(v_{k}\right)>x_{1} \cos (\theta / 2)$ : (2) Separated Form.

The function $R(v)$ in Eq. (17c) was assumed to be constant in the saddle-point integration and is given explicitly by

$$
R(v)=\frac{\left(\varrho_{1} / \varrho_{2}\right) x_{t}^{2} D_{1}(v)+i x_{1} \sin \alpha D_{2}(v)}{\left(\varrho_{1} / \varrho_{2}\right) x_{t}^{2} D_{1}(v)-i x_{1} \sin \alpha D_{2}(v)},
$$

where $D_{1}, D_{2}$ are the respective $2 \times 2$ minor determinants obtained by expanding $D(v)$ by its first column.

By the use of Eq. (8), Eq. (17b) can be written for $r \rightarrow \infty$ (the asymptotic approximation for $r \rightarrow \infty$ has already been made in Eq. (17c)) as

$$
\begin{aligned}
p_{\mathrm{c}} \cong- & \left(\frac{8 \pi}{k_{1} r}\right)^{1 / 2} e^{i\left(k_{1} r-\pi / 4\right)} \\
& \times \sum_{k=1}^{\infty} \frac{1}{\sin \pi v_{\mathrm{k}}} \frac{B\left(v_{\mathrm{k}}\right)}{\dot{D}\left(v_{\mathrm{k}}\right)}\left\{\begin{array}{l}
\cos \left(v_{k} \theta\right) e^{-i \pi v_{k}} \\
\cos v_{k}(\pi-\theta)
\end{array}\right\} \text { (2) }
\end{aligned}
$$

At this point it is possible to demonstrate explicitly the circumferential character of the creeping waves. For the unseparated form (1) in Eq. (20) we rewrite the trigonometric terms

$$
\frac{\cos \left(\nu_{\mathrm{k}} \theta\right)}{\sin \left(\pi v_{\mathrm{k}}\right)}=-i \sum_{\lambda= \pm 1} \sum_{m=0}^{\infty} e^{i v_{k}(\lambda \theta+\pi+2 m \pi)},
$$

which is uniformly convergent for $\operatorname{Im}\left(v_{k}\right)>0$. Inserting the time dependence $\exp (-i \omega t)$ we see that the residue series (20) is made up of terms having the form

$$
e^{i\left( \pm v_{k} \theta-\omega t\right)}=e^{-\operatorname{Im}\left(v_{k}\right)( \pm \theta)} e^{i\left[ \pm \operatorname{Re}\left(v_{k}\right) \theta-\omega t\right]},
$$


which are clearly circumferential waves travelling in the $\pm \theta$ directions, and are damped with an attenuation factor proportional to $\operatorname{Im}\left(v_{k}\right)$. Their phase velocities are given by

$$
c_{k}^{\mathrm{ph}}=\frac{\omega a}{\operatorname{Re}\left(v_{\mathrm{k}}\right)}=\frac{x_{1}}{\operatorname{Re}\left(v_{\mathrm{k}}\right)} c_{1} .
$$

For pulses we can also define the group velocities

$$
c_{k}^{\mathrm{gr}}=\frac{\mathrm{d} x_{1}}{\mathrm{~d} \operatorname{Re}\left(\nu_{\mathrm{k}}\right)} c_{1} .
$$

The additional summation over $m$ in Eq. (21) represents waves which have circumnavigated the cylinder $m$ times. Equation (22) also shows that no second or fourth quadrant zeroes $v_{k}$ are allowed since these would lead to physically unacceptable exponentially increasing waves. This was one of the main factors for determining the contours in Fig. 2.

The differential scattering cross section, Eq. (7), is now given by (approximately, because we have neglegted the background integral $(12 \mathrm{c}))$

$$
(\mathrm{d} \sigma / \mathrm{d} \theta) \cong \lim _{r \rightarrow \infty} r\left|p_{\mathrm{g}}+p_{\mathrm{c}}\right|^{2},
$$

which by using Eqs. (17c) and (20) can be put in the form

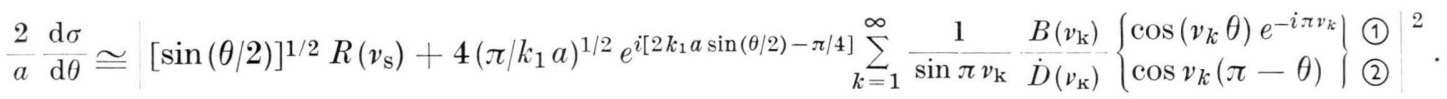

This is much more complicated than the relatively simple expression in Eq. (9). However, whereas the latter may need on the order of 1000 terms before starting to converge, we found that for $k_{1} a \gtrsim 5$ we need sum only three or four terms (the first-quadrant Rayleigh poles plus at most two Franz poles) in Eq. (26) to obtain four-digit accuracy. A numerical evaluation of Eq. (26) for an aluminium cylinder is shown in Fig. 11. More extensive computations for aluminium shells can be found in Refs. ${ }^{14}$ and 16 .

Finally we consider the paths of the creeping waves around the cylinder, which determines the critical angles at which a creeping wave may be launched on the cylinder and radiate off to an observer after $m$ circumnavigations. These critical angles have been derived intuitively in KELLER's 22 "geometrical theory of diffraction" by noting that at these angles there exists a resonance effect: the incident wave velocity is equal to the component in the direction of incidence of the creeping-wave velocity. In Ref. 13 we have established them rigorously by following the path of a delta-function pulse and correlating its travel times with causality requirements. The picture which emerges from such an analysis is given in Fig. 4. Figure 4 a shows a creeping wave (solid line) launched on the cylinder at the critical angle $\alpha$ (measured from the shadow boundary), then proceeding to the observer $\mathrm{P}$, leaving the cylinder at the same critical angle $\alpha$ (measured from $\mathrm{N}$ - the normal to the observation direction). The rigorous theory 13 predicts these

22 B. R. Levy and J. B. Keller, Commun. Pure Appl. Math. 12, 159 [1959].

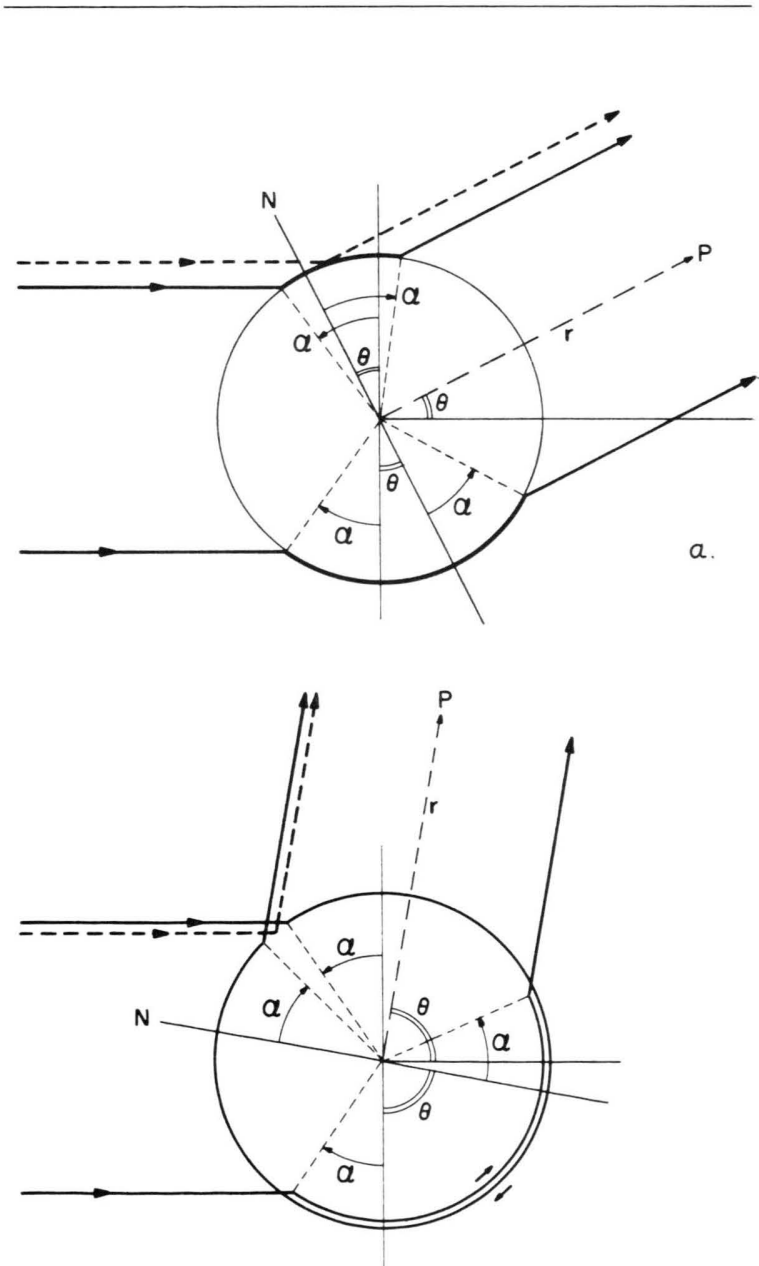

Fig. 4. Geometry of the Circumferential Waves: (a) $\theta<2 \alpha$ corresponding to the "Unseparated Form"; (b) $\theta>2 \alpha$ corresponding to the "Separated Form". The geometrically reflected wave is indicated by the dashed line. 



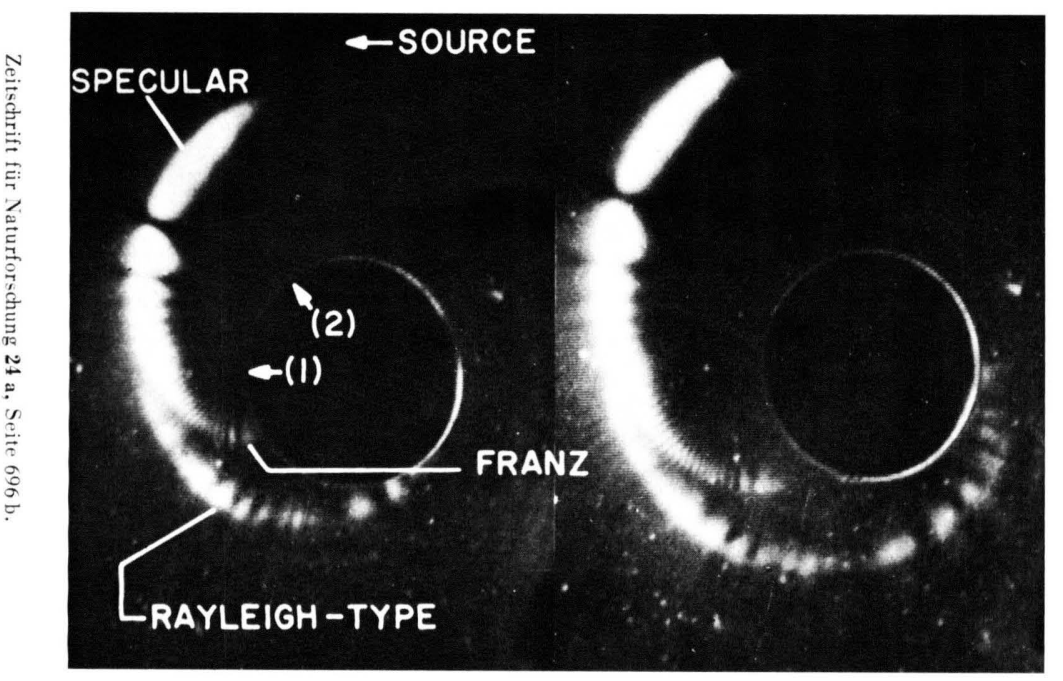

Fig. 5. Schlieren photographs of the wavefronts resulting from Franz- and Rayleigh-type circumferential waves on an aluminum cylinder in water $\left(x_{1}=323.7\right)$. The wavefronts in (b) occur at a later time than those in (a).

Fig. 7. Schlieren photograph which is a double exposure showing two successive positions of the radiated wave resulting from the circumferential wave with approximately a $15^{\circ}$ incidence and emergence angle $\left(x_{1}=1008, t_{2}>t_{1}\right)$.

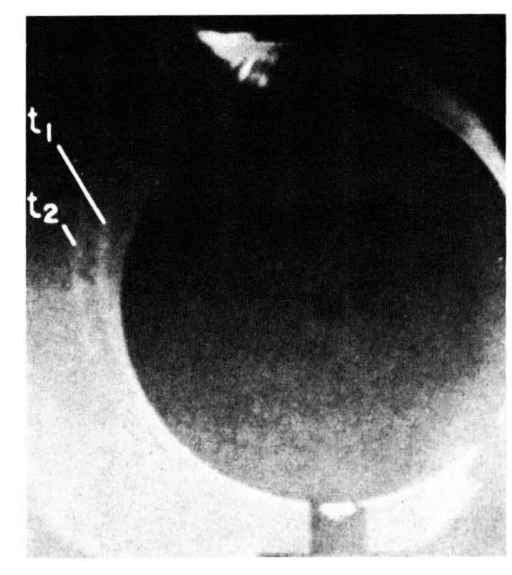

Fig. 8. (a) Schlieren photograph of the waves produced when the upper right quarant is insonified with an incident plane wave traveling toward the bottom of the picture. (b) Diagram for interpretation of photograph in (a): A - Illuminated portion; B - Specular reflection; C - Franz wave; D - 30 wave; E - Through compressional wave.

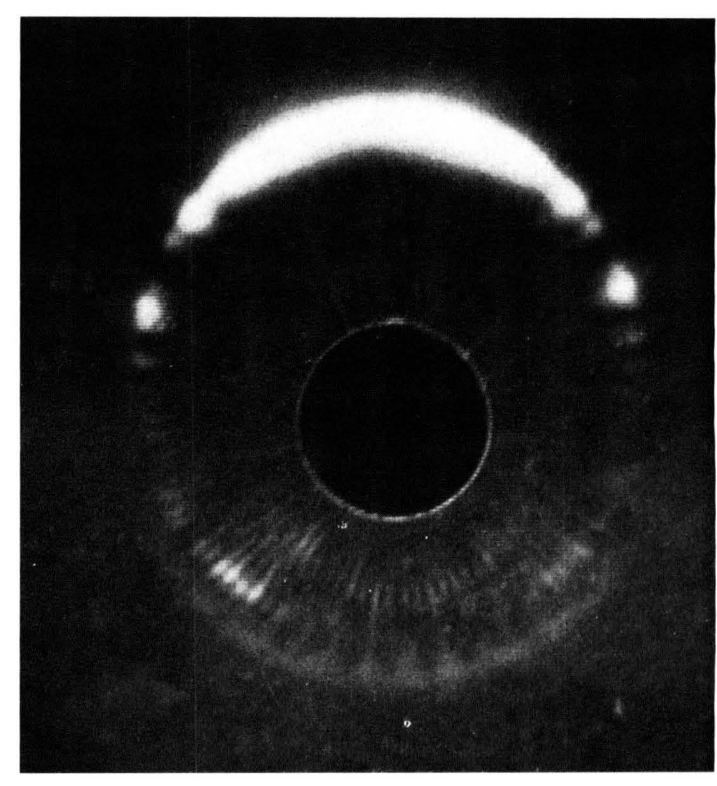

Fig. 9. Schlieren photograph of the entire diffracted field produced when the upper half of the cylinder is insonified. 
angles to be

$$
\begin{aligned}
\alpha_{k}^{\mathrm{ph}} & =\lim _{s \rightarrow \infty} \cos ^{-1}\left(v_{k} / x_{1}\right) \\
& \cong \cos ^{-1}\left(c_{1} / c_{k}^{\mathrm{ph}}\right) \quad \text { for } \mathrm{C} . \mathrm{W} ., \\
\alpha_{k}^{\mathrm{gr}} & =\lim _{s \rightarrow \infty} \cos ^{-1}\left(\mathrm{~d} v_{k} / \mathrm{d} x_{1}\right) \\
& \cong \cos ^{-1}\left(c_{1} / c_{k}^{\mathrm{gr}}\right) \quad \text { for pulses },
\end{aligned}
$$

where $s$ is the integration variable in a Laplace transformation used to obtain a delta-function pulse from the plane wave. These angles in general are complex (except for the rigid or soft cylinder in which case they become $\alpha_{k}=0$, showing that the Franz waves are launched at the shadow boundary). The approximation of taking their real parts (the right-hand sides of Eqs. (27)) gives meaningful physical angles if $\operatorname{Im}\left(v_{k}\right)$ is small, which is the case for all Rayleigh-type zeroes. For $\theta=2 \alpha$ the distance which the upper creeping wave in Fig. 4 a has to travel on the cylinder shrinks to zero. It therefore has to make a full revolution around the cylinder before proceeding to $\mathrm{P}$, as shown in Fig. $4 \mathrm{~b}$. (This means that $m$ has increased by one in Eq. (21)). The condition $\theta=2 \alpha$ is precisely the same as that in Eq. (18) for the change-over from the unseparated to the separated forms for the residue contributions. At this point the saddle point (15) is directly under one of the Rayleigh poles. The separated form of the solution (which is to be used for $\theta>2 \alpha$ ) predicts exactly, from our causality arguments ${ }^{13}$, the path shown in Fig. $4 \mathrm{~b}$ in agreement with physical intuition.

\section{Part B: Experiment}

\section{Schlieren Observation}

Distinct circumferential waves are related to poles or groups of poles in the complex $v$-plane described in the theory. Each of three of these waves may be separately generated by acoustic pulse illumination of a selected small portion of a cylinder with a narrow-beam acoustic source directed at a specific incidence angle. The radiation into the field around the cylinder resulting from the circumferentially traveling waves may be observed directly by means of Schlieren visualization. All the observations referred to here are for aluminium (type

* Fig. 5, 7-9 on p. 696 b.
$6061)$ cylinders immersed in water. The simultaneous generation of two distinct types of circumferential waves is shown in Fig. $5 *$. Figure $5 \mathrm{~b}$ is taken at a later time than Fig. 5a showing the progression of the radiated waves. The source transducer is at the top of the photograph radiating downward. The part of the incident beam striking the cylinder cross section tangentially, generates the Franz wave. If only the point of tangential incidence (labeled (1) in Fig. 5a) is illuminated, the Franz wave alone would be generated ${ }^{18}$. Most of the wavefront containing the Franz-wave radiation is specular reflection from the cylinder surface. Only the portion to the right of a vertical line, from the transducer face through the tangential point of incidence, results from the Franz wave. This may be considered a diffracted circumferential wave. In Fig. 5, the source beam was sufficiently wide that a Rayleigh-type wave was also generated in the neighborhood of the point labeled (2) on the circumference in Fig. $5 \mathrm{a}$. This wave has also been generated and observed separately ${ }^{18,23}$. A diagram intended to aid in the interpretation of this wave is shown in Fig. 6. An incident ray generates the normally expected specular reflection at an anple $\varphi$. This incident and specular angle is related to the critical angle $\alpha$ in Part A by $\varphi=(\pi / 2)-\alpha$. On striking the cylinder, a circumferential wave is generated which travels inside the cylinder boundary, radiating part of its energy out of the cylinder at an angle as it circum-

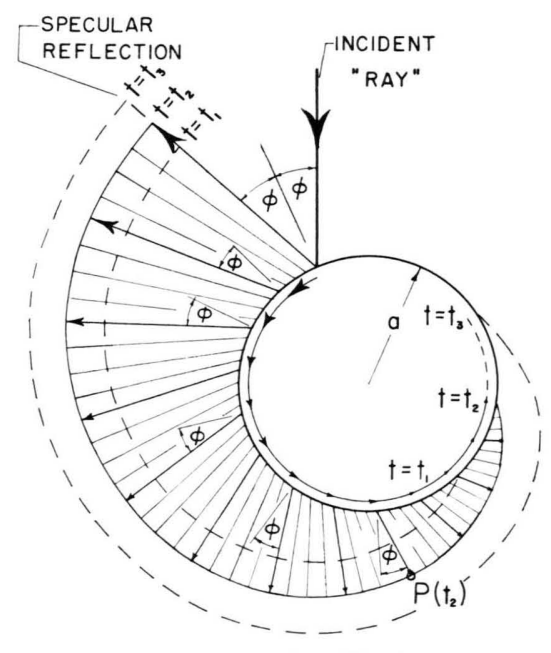

Fig. 6. A geometric construction illustrating circumferential wavefront propagation.

23 R. R. Goodman, R. E. Bunney, and S. W. Marshall, J. Acoust. Soc. Am. 42, 523(L) [1967]. 
navigates the cylinder. This generates a wavefront labeled at three different times $t_{1}, t_{2}$, and $t_{3}$, which correspond to the Schlieren visualizations. The decreasing arrow head sizes along the circumferential path and along the wavefront indicate the magnitude of the pressure amplitude. This is thus an attenuating wave and may be regarded as a refracted circumferential wave. A hydrophone placed in the field on the side of the cylinder opposite from the source, at a point such as $\mathrm{P}$, would detect the passage of the radiated pressure front. From hydrophone measurements, at a point such as $\mathrm{P}$ and Schlieren photographs at different times, the properties of the circumferential wave may be interpreted. Experimental isolation and measurements of these waves have been carried out for a range of $x_{1}=k_{1} a$ from 54 to 1008. Such experiments have resulted in the following conclusions about the Franz and Rayleightype waves. The Franz wave is primarily related to the geometric form or curvature of the surface on which it travels. It propagates on the outside of the boundary at a circumferential speed $\left(c_{\mathrm{c}}\right)$ only $3 \%$ less (on aluminium) than the free water-borne wave $\left(c_{\mathrm{W}}\right)$ at $x_{1}$ of 54 . The difference from $c_{\mathrm{W}}$ decreases to $1 \%$ above an $x_{1}$ of 150 . The circumferential velocity may be predicted to within $\pm 0.5 \%$ by the formulation for the rigid cylinder of Franz ${ }^{4}$ above $x_{1}=54$. The attenuation of the Franz wave is, however, significantly affected by the material of the cylinder. Its magnitude was measured to be between 2 and $3 \mathrm{~Np} / \mathrm{rad}$ at these high values of $x_{1}$ which is significantly less than that predicted by the formulation by Franz $^{4}$ for the rigid cylinder.

The Rayleigh-type wave is primarily related to the material of the diffracting body and is a surface wave propagating on the inside of the boundary. Its speed (for aluminium, at high $x_{1}$ ) is approximately 2.5 times $c_{\mathrm{w}}$. Its attenuation, which is considerably less than that of the Franz wave, ranges between 0.10 and $0.18 \mathrm{~Np} / \mathrm{rad}$ for large $x_{1}$. The incidence and emergence angle for the Rayleightype wave was measured to be approximately $30^{\circ}$ for aluminium at high $x_{1}$. The approximate theoretical formulation comparable to Eq. (27) in Part A is $\varphi=\sin ^{-1}\left(c_{\mathrm{c}} / c_{\mathrm{w}}\right)$. Measured velocities predict a value of $\varphi$ of $23^{\circ}$ for an $x_{1}$ of 323.7 . This is considered reasonable agreement between theory and experiment considering the difficulty of measurement of acoustic-beam angle direction relative to a curved surface.
A third wave, also a refracted circumferential wave was found to be generated at a value of $\varphi$ of approximately $15^{\circ}$ for large $x_{1}$. The radiation resulting from such a wave is shown in Fig. 7 for an $x_{1}$ of 1008. Here the wavefront is shown at two different times by means of a double exposure $\left(t_{2}>t_{1}\right)$. Figure 6 also describes the behavior of this wave. Its circumferential speed is approximately $6.5 c_{\mathrm{w}}$. Although it is generated to a lesser degree than the wave generated at $30^{\circ}$ incidence, its attenuation was measured to be approximately $20 \%$ lower for large $x_{1}$. The degree to which a wave is generated is determined by the residue at the pole related to that wave, viz. the corresponding $k$-th term in Eq. $(17 \mathrm{~b})$.

Simultaneous insonification of all incidence angles is accomplished by causing an acoustic pulse to fall incident on one quadrant of the cylinder cross section. In such a case, shown in Fig. 8, all possible types of waves are generated. Figure $8 \mathrm{~b}$ indicates the meaning of the various features of Fig. $8 \mathrm{a}$. In Fig. 8, the pulse falling incident on the upper right quadrant $[\mathrm{A}]$ generates the specular reflection [B] and the Franz wave [C]. At the value of $x_{1}$ of 215.8 for this case, the Shear wave transmitted through the body of the cylinder emerges mixed with the Rayleigh-type circumferential wave [D]. Since the circumferential wave occurring at $15^{\circ}$ incidence is generated to such a smaller degree than the others, it is not apparent in this picture. However, radiaton $[\mathrm{E}]$, as a result of the compressional wave transmitted through the body of the cylinder, is seen.

When the entire cylinder was insonified from above with an incident wave which was essentially plane, the Schlieren photograph of Fig. 9 resulted. Here sets of circumferential waves are generated in both directions around the cylinder causing interference between them, resulting in a somewhat confused picture. However, not so confused that the reader cannot select identifiable portions of wavefronts with far less doubt than he would have had without the benefit of the pictures of the separated waves. A hydrophone placed at a point of the field on the shadow side of the cylinder would produce a very complicated pulse sequence of much longer total duration than the incident pulse.

\section{I1. Hydrophone Detection of a Pressure Pulse}

The configuration for an experiment which utilized a hydrophone on the side of the cylinder opposite from the source is shown in Fig. 10a for 


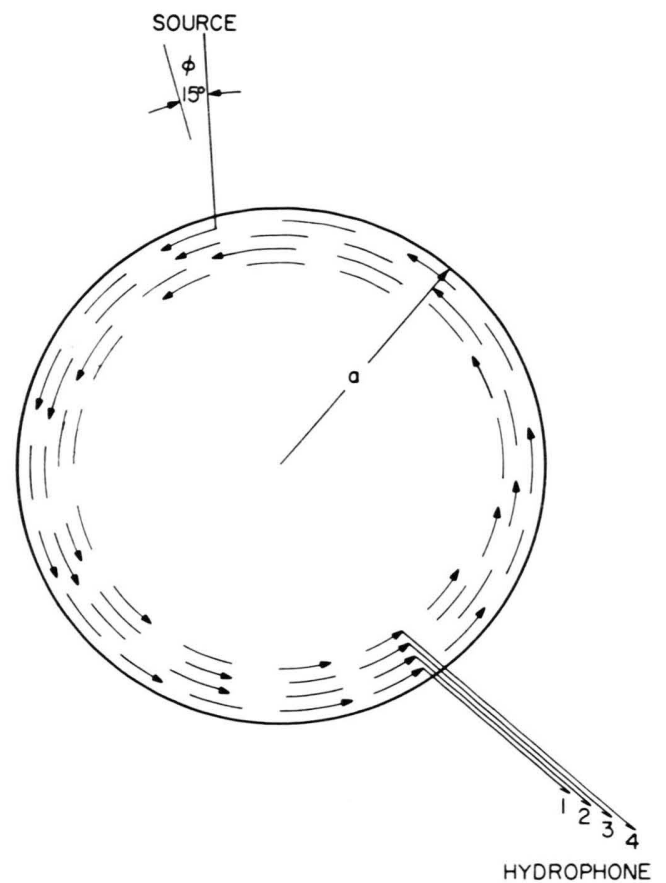

(a)

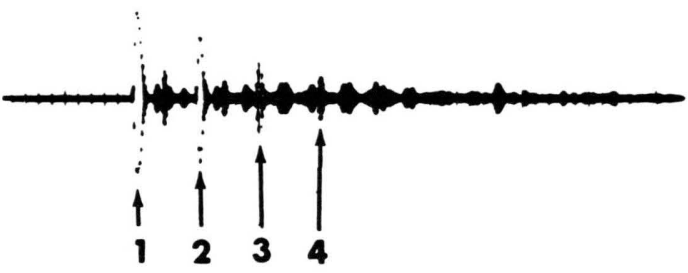

(b)

Fig. 10. (a) Diagram of the experimental arrangement and pulse path for detecting circumferential-wave pulses radiated from a cylinder and (b) the resulting pressure-pulse sequence received at the hydrophone.

the wave generated at $15^{\circ}$ incidence. The circumferential wave is generated upon incidence and travels around the inside of the boundary. The wave path is shown in Fig. 10a as a spiral to avoid confusion. Actually, the path is identical on successive circuits as are the paths to the hydrophone. The wave radiates to the hydrophone after part of a circuit. Part of the wave continues around the circumference of the cylinder and after a full circuit is radiated to the hydrophone a second time, and so on. The resulting pulse sequence at the hydrophone is shown in Fig. 10b. The labels on the pulses indicate the sequence of reception, in time. It can be seen that a background of other pulses is present, probably relating to other poles of the Rayleigh-type as theory indicates should exist at large values of $x_{1}$. From such pulse sequences shown in Fig. 10 b, the velocity and attenuation of these waves can be determined.

The degree to which the creeping-wave formulation is a meaningful description of the diffracted field is illustrated by a comparison of experimentally measured values of differential scattering cross section versus $x_{1}$. An example of such a comparison is shown in Fig. 11 for aluminium cylinders for values of $x_{1}$ between 5 and 25 . The reasonable correspondence between theory and experiment demonstrates that the creeping wave formulation is a useful and realistic description of the actual scattered field.

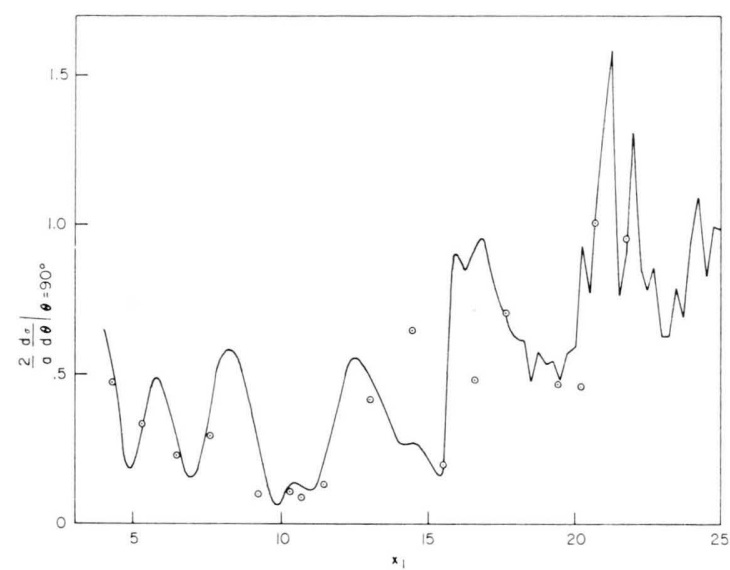

Fig. 11. Experimentally measured values of differential scattering cross section plotted with a theoretical curve computed by the creeping-wave formulation.

\section{Conclusion}

The application of the Sommerfeld-Watson transformation to the classical normal-mode series solution of the wave equation for cylindrical geometry, permits the recognition of waves which travel circumferentially on the cylinder boundary. When the cylinder is elastic, two types of waves which have circumferential paths are found to contribute to the diffraction caused by the cylinder. One is the Franztype wave known to also be present in the extreme cases of "hard" and "soft" boundary conditions. The other, called a Rayleigh-type wave is present because of the elasticity of the cylinder. 
Experimentally, three separate predominant circumferential waves have been observed on elastic cylinders. The waves may be separately observed by using short-pulse narrow-beam acoustic signals.

The "Franz" wave is generated at tangential incidence and travels on the external side of the cylinder surface. Its properties are primarily determined by the geometry of the diffracting or reflecting object, although the elastic properties of the cylinder do influence the properties of the wave, especially the attenuation. The creeping wave is highly attenuated, in the order of $3 \mathrm{~Np} / \mathrm{rad}$. The velocity for $k a$ 's greater than 54 is only $1 \%$ lower than that of the free water-borne wave and may be computed to within less than $\pm 0.5 \%$ by Franz's formula for the rigid case.

A refracted circumferential wave is characteristically generated at an incidence and emergence angle near $30^{\circ}$, travels on the inside of the cylinder boundary, and is less attenuated than the Franz wave. Its speed is near 2.5 times the free water-borne wave speed. The wave properties are more directly related to the elastic properties of the surface than the curvature of the surface on which it travels. This wave has been reasonably identified as one whose properties approach the properties of the Rayleigh wave for a plane surface. It is, therefore, referred to as a "Rayleigh-type" wave.

A third wave was observed which is very similar to the one generated at $30^{\circ}$ incidence except that it is generated at an incidence and emergence angle of $15^{\circ}$. It also travels on the inside of the curved boundary. It is the least attenuated of the three distinct waves observed and is apparently generated to a lesser degree than the other two. Its speed is much higher than the other two; 6.5 times the free water-borne-wave speed. This wave may be related to one of the higher Rayleigh-mode poles in the complex $v$-plane.

The three types of waves were measured using pulses. Values of $x_{1}$ or $k a$ ranged from 54 to 1008 with two independent experimental methods. A pressure-pulse sequence was measured with a hydrophone and radiated wavefronts were observed with the Schlieren method of wavefront visualization. The two methods established the same velocities stated above for the refracted circumferential waves. Experimental agreement is found with the total field calculation in the form of differential scattering cross section over a $k a$ range between 5 and 25 .

Thus, the properties of three distinct circumferential waves on elastic cylinders are established. Not only is the so-called creeping-wave solution useful since it converges rapidly, facilitating computation, but also the awareness of separate circumferential waves gives an insight into the diffraction phenomenon.

\section{Acknowledgements}

The authors acknowledge the support of the Office of Naval Research through the Interdisciplinary Acoustics Program at The Catholic University of America, and the Naval Research Laboratory and Naval Weapons Laboratory. 